\title{
A GENERALIZATION OF THE MIKUSINSKI OPERATIONAL CALCULUS
}

\author{
JAMES A. WOOD 1
}

0. Introduction. In his version of the operational calculus Mikusinski uses as a starting point the familiar theorem from algebra that every integral domain can be embedded isomorphically in a field. He shows that the class of complex-valued continuous functions defined on $[0, \infty)$ forms an integral domain when addition and multiplication are taken to be pointwise addition and convolution of functions, respectively. The resulting field is called the field of Mikusinski operators.

In the present paper we generalize Mikusinski's basic results by developing an operational calculus for strongly continuous functions defined on $[0, \infty)$ and having values in any Banach algebra with unit element. The Mikusinski operational calculus will then be a special case of our theory, obtained by taking the Banach algebra to be the complex numbers.

The paper is divided into four parts. The first gives an algebraic construction; the second shows how the algebraic construction may be used to develop the operational calculus mentioned above; the third indicates some basic results which carry over from the (Mikusinski) operational calculus to this more general setting. Finally, in the last section, we generalize within the framework of the operational calculus a recent theorem of T. K. Boehme.

We assume the reader is familiar with the basic notations and results in the books by Mikusinski [3] and Erdélyi [2].

1. An algebraic construction. Let $R$ be a ring and $D$ a fixed nonempty subset of $R$ subject to the following two conditions:

(i) if $x \in D$, then $x y=y x$ for all $y \in R$,

(ii) if $x \in D$, the equation $x y=0, y \in R$ implies $y=0$.

It is clear that $D$ is a multiplicative semigroup and does not contain the additive identity in $R$.

Now consider the set of ordered pairs $(x, a)$ where $x \in R, a \in D$. We say that $(x, a)=(y, b)$ iff $x b=y a$. We make the convention of writing an ordered pair as a fraction. Thus for the ordered pair $(x, a)$ we

Received by the editors November 28, 1966.

${ }^{1}$ ACKNowledgement. The results of this paper were part of the author's doctoral dissertation at the University of Virginia. The author wishes to thank Professor M. Rosenblum for suggesting the problem to him. 
write $x / a$. It is easy to check that the above notion of equality is an equivalence relation. If one defines addition by $x / a+y / b=(x b+a y) / a b$ and multiplication by $(x / a)(y / b)=x y / a b$, it is also straightforward to check that the operations are well defined and that, in fact, the equivalence classes of ordered pairs together with these two operations form a ring $R^{*}$. $R$ is isomorphic to the subring of $R^{*}$ consisting of all fractions of the form $a x / a, a \in D, x \in R . a / a$ is the unit in $R^{*}$.

One notes that if the ring $R$ is an integral domain and one takes the semigroup $D$ to be $R-\{0\}$ then the above construction reduces to the classical construction for embedding an integral domain in a field. Since $R^{*}$ has a unit one might expect in general that there are some fractions in $R^{*}$ which have inverses. (By an inverse we mean both a left and right inverse.) The following theorem gives a necessary and sufficient condition that a fraction have an inverse.

THEOREM 1.1. The fraction $x / a \in R^{*}$ has an inverse iff there exists a $y \in R$ and $\alpha \in D$ such that $x y=y x=\alpha$.

Proof. Suppose $x / a$ has an inverse $y / b$. Then $x y / a b=y x / b a=c / c$ and this means $x y c=a b c$ and $y x c=b a c$; i.e. $x y=y x=a b=\alpha$. Conversely, suppose there is a $y \in R$ and $\alpha \in D$ such that $x y=y x=\alpha$. Then the fraction $a y / \alpha$ is the inverse of $x / a$ since $x / a \cdot a y / \alpha=x y / \alpha=\alpha / \alpha$ $=a y / \alpha \cdot x / a$.

2. Operators. We now generalize the Mikusinski operational calculus using the algebraic construction in $\S 1$. Let $\mathrm{B}$ be a Banach algebra with unit and let $\mathrm{S}$ be the set of all strongly continuous functions on $[0, \infty)$ having values in $B$, together with the operations of addition and multiplication defined as follows:

$$
\text { (i) } \mathrm{f}+\mathrm{g}(x)=\mathrm{f}(x)+\mathrm{g}(x), \quad \text { (ii) } \mathrm{f} * \mathrm{~g}(x)=\int_{0}^{x} \mathrm{f}(x-t) \mathrm{g}(t) d t \text {. }
$$

The integral defining convolution is, of course, the Bochner integral and one can easily check that $\mathrm{S}$ is a ring. Let us now denote by $S$ the set of functions of the form $u \cdot \mathrm{f}(x)$ where $u$ is the unit in the Banach algebra and $\mathrm{f}(x)$ is a complex-valued continuous function not identically zero. Functions belonging to $S$ will be denoted by italic letters. Clearly, $S$ is a subset of $\mathrm{S}$. Moreover, we have the following theorem.

Theorem 2.1. (i) If $f \in S$ then for any $g \in \mathrm{S}, \mathrm{g} * f=f * \mathrm{~g}$. (ii) If $f \in S$ and $f * \mathrm{~g}(x)=0$ for all $x$, where $\mathrm{g} \in \mathrm{S}$, then $\mathrm{g}(x)=0$ for all $x$.

Proof. If $\mathrm{B}^{*}$ denotes the set of bounded linear functionals on $\mathrm{B}$ then for any $l \in B^{*}$ we have 


$$
\begin{aligned}
l[f * \mathrm{~g}(x)] & =l\left[\int_{0}^{x} f(x-t) \mathrm{g}(t) d t\right]=\int_{0}^{x} f(x-t) l[\mathrm{~g}(t)] d t \\
& =\int_{0}^{x} l[\mathrm{~g}(x-t)] f(t) d t=\int_{0}^{x} l[\mathrm{~g}(x-t)] f(t) d t \\
& =l\left[\int_{0}^{x} \mathrm{~g}(x-t) f(t) d t\right]=l[\mathrm{~g} * f(x)] .
\end{aligned}
$$

Hence $f * \mathrm{~g}=\mathrm{g} * f$.

Assume now that $f * \mathrm{~g}(x)=0$ for all $x$. Then for any $l \in \mathrm{B}^{*}$, $l[f * \mathrm{~g}(x)]=\int_{0}^{x} f(x-t) l[\mathrm{~g}(t)] d t=0$. But by Titchmarsh's theorem (Mikusinski [3, pp. 15-23]) this implies $l[\mathrm{~g}(t)]=0$ for all $t \geqq 0$. Since this is true for every $l \in \mathrm{B}^{*}$, we conclude that $\mathrm{g}(t) \equiv 0$ for all $t \geqq 0$.

It now follows from the construction of $\S 1$ (taking $R=\mathrm{S}$ and $D=S$ ) that fractions of the form $\phi / f, \phi \in \mathrm{S}, f \in S$ form a ring $\mathrm{S}^{*}$ which contains $\mathrm{S}$ isomorphically. Moreover, $\mathrm{S}^{*}$ has a unit and it follows from Theorem 1.1 that a necessary and sufficient condition that a fraction $\phi / f \in S^{*}$ have an inverse is that there exist a function $\psi \in S$ and a function $\alpha \in S$ such that $\phi * \psi=\psi * \phi=\alpha$. We follow Mikusinski's convention of writing a function in braces; thus $\{u\}$ means the function whose value at each $t \geqq 0$ is the unit in $B$.

One notes also that $\mathrm{S}^{*}$ can be made into an algebra by defining $\alpha\{\boldsymbol{\phi}(t)\} /\{f(t)\}=\{\alpha \boldsymbol{\phi}(t)\} /\{f(t)\}$, where $\alpha$ is a complex number. Also, in analogy with the classical case, it is possible to identify certain subsets of $\mathrm{S}^{*}$ with (i) the algebra of complex numbers; (ii) the Banach algebra B; (iii) the locally (Bochner) integrable functions; (iv) the algebra S. For example, one can identify $B$ and the subalgebra of all fractions of the form $\{\mathrm{a}\} /\{u\}, \mathrm{a} \in \mathrm{B}$. According to current convention, if $\mathrm{a} \in \mathrm{B}$ then we use the same symbol a for both $\{\mathrm{a}\} /\{u\}$ and $\mathrm{a}$. A similar convention holds for the other cases. Following the terminology of Mikusinski, we shall call the members of $\mathrm{S}^{*}$ operators. Finally, note that in general this operational calculus is not commutative.

3. Elementary operators. As in the classical case, one can define the integral and differential operators.

Definition 3.1. If $u$ is the unit in the Banach algebra, then $l=\{u\}$ is called the integral operator and $l^{-1}=s$ is called the differential operator. $l$ is called the integral operator because if $\{\mathrm{f}(t)\} \in \mathrm{S}$, then $l\{\mathrm{f}(t)\}=\left\{\int_{0}^{t} \mathrm{f}(\tau) d \tau\right\} . s$ is called the differential operator because of the following:

THEOREM 3.1. If the function $\{\mathrm{f}(t)\} \in \mathrm{S}$ has a strong derivative $\left\{\mathrm{f}^{\prime}(t)\right\} \in \mathrm{S}$, then $s\{\mathrm{f}(t)\}=\left\{\mathrm{f}^{\prime}(t)\right\}+\mathrm{f}(0)$. 
Proof. The proof is the same as the classical case.

Clearly, one can generalize by saying that if $\{\mathrm{f}(t)\} \in \mathrm{S}$ has $n$ strong derivatives all belonging to $\mathrm{S}$ then $s^{n}\{\mathrm{f}(t)\}=\left\{\mathrm{f}^{(n)}(t)\right\}+\mathrm{f}^{(n-1)}(0)$ $+s^{(n-2)}(0)+\cdots+s^{n-1} f(0)$.

If $\mathrm{a}$ is a fixed element in the Banach algebra and $e^{\mathrm{a} t}$ is defined by its power series expansion, then one can prove using Theorem 3.1 that $(s-\mathrm{a})^{-1}=\left\{e^{\mathrm{a} t}\right\}$, and this gives the connection between the differential operator and the exponential function. All the elementary formulae from the Mikusinski operational calculus can be generalized to the present setting. For example, under the conditions that $\mathrm{a}, \mathrm{b} \in \mathrm{B}$, $\mathrm{b}^{-1}$ exists and $\mathrm{a}$ and $\mathrm{b}$ commute we have $\left[(s-\mathrm{a})^{2}+\mathrm{b}^{2}\right]^{-1}=\left\{\mathrm{b}^{-1} e^{\mathrm{a} t} \sin \mathrm{b} t\right\}$.

It is also clear that one may use the operational calculus as Mikusinski does to solve certain types of differential equations. For example, consider the equation $\mathrm{a}\left\{\mathrm{f}^{\prime \prime}(t)\right\}+\mathrm{b}\left\{\mathrm{f}^{\prime}(t)\right\}=\{\mathrm{g}(t)\}, \mathrm{f}(0)=A$, $\mathrm{f}^{\prime}(0)=B, \mathrm{a}, \mathrm{b} \in \mathrm{B}, \mathrm{a}^{-1}$ exists and $\{\mathrm{g}(t)\} \in \mathrm{S}$. The operational form of the equation is a $\left[s^{2}\{\mathrm{f}(t)\}-B-s A\right]+\mathrm{b}[s\{\mathrm{f}(t)\}-A]=\{\mathrm{g}(t)\}$. Solving the equation for $\{\mathrm{f}(t)\}$ one obtains

$$
\begin{aligned}
\{\mathrm{f}(t)\}= & \left\{\int_{0}^{t} e^{-\mathrm{a}^{-1} \mathrm{~b}(t-\tau)} B d \tau\right\}+\left\{e^{-\mathrm{a}^{-1} \mathrm{~b} t} A\right\} \\
& +\left\{\int_{0}^{t} e^{-\mathrm{a}^{-1} \mathrm{~b}(t-\tau)} \mathrm{a}^{-1} \mathrm{~b} A d \tau\right\}+\left\{\int_{0}^{t} e^{-\mathrm{a}^{-1} \mathrm{~b}(t-\tau)} \mathrm{a}^{-1} \int_{0}^{\tau} \mathrm{g}(u) d u\right\} .
\end{aligned}
$$

4. An application. In 1965, T. K. Boehme proved the following theorem. (See Boehme [1].)

ThEOREM (BOEHME). Let $\left\{\mathrm{g}_{n}\right\}, n=1,2,3, \cdots$ be a sequence of complex-valued continuous functions on $[0, \infty)$. A necessary and sufficient condition that there exists a sequence $\left\{\mathrm{f}_{n}\right\}$ (complex-valued and continuous) and a function $\mathrm{g}$ also complex-valued and continuous such that $\mathrm{g}=\mathrm{g}_{n} * \mathrm{f}_{n}$ for $n=1,2,3, \cdots$ is that there exist an interval $[0, T]$ such that for no $n$ does $\mathrm{g}_{n}$ vanish identically on $[0, T]$.

One could ask whether there is an analogue of the theorem for strongly continuous functions having values in the Banach algebra $B$. The operational calculus provides a clue to the solution. It turns out that if each $g_{n}$ has an inverse (as an operator) and satisfies a certain auxiliary condition then one can prove a generalization of Boehme's theorem. Before stating the theorem, we need a definition.

DEFinition 4.1. Suppose $\mathrm{f} \in \mathrm{S}$. If $\mathrm{f}$ has an inverse as an operator then there is a function $\mathrm{g} \in \mathrm{S}$ and a function $h \in S$ such that $\mathrm{f} * \mathrm{~g}$ $=\mathrm{g} * \mathrm{f}=h$. (Note that $\mathrm{g}$ and $h$ need not be unique.) We say that $\mathrm{f}$ belongs to $S_{T} \subset S$ if one can find at least one pair of functions $g \in S$, 
$h \in S$ such that $\mathrm{f} * \mathrm{~g}=\mathrm{g} * \mathrm{f}=h$ and $h$ does not vanish identically on the interval $[0, \mathrm{~T}]$.

We then have the following theorem.

Theorem 4.1. Let $\left\{\mathrm{G}_{n}\right\}, n=1,2,3, \cdots$ be a sequence of functions belonging to S. A necessary and sufficient condition that there exists a sequence of functions $\left\{\mathrm{F}_{n}\right\}$ in $\mathrm{S}$ and a function $G \in S$ such that $G=\mathrm{F}_{n}$ * $\mathrm{G}_{n}=\mathrm{G}_{n} * \mathrm{~F}_{n}$ for $n=1,2,3, \cdots$ is that there exist a $\mathrm{T}>0$ such that for all $n, \mathrm{G}_{n} \in \mathrm{S}_{T}$.

Proof. The necessity is clear. Let us therefore suppose that $\left\{G_{n}\right\}$ is a sequence of functions in $S$ and that there is a $T>0$ such that for all $n, \mathrm{G}_{n} \in \mathrm{S}_{T}$. By the definition of $\mathrm{S}_{T}$, for each $n$ there is a function $\mathrm{H}_{n}$ and a function $h_{n}$ such that $\mathrm{G}_{n} * \mathrm{H}_{n}=\mathrm{H}_{n} * \mathrm{G}_{n}=h_{n}$ and none of the $h_{n}$ vanish identically on the interval $[0, T]$. Then by Boehme's theorem there exists a sequence of complex-valued continuous functions $\left\{\mathrm{f}_{n}\right\}$ and a complex-valued continuous function $G$ such that $\mathrm{f}_{n} * h_{n}$ $=G$. From this it follows that $\mathrm{G}_{n} *\left(\mathrm{H}_{n} * \mathrm{f}_{n}\right)=h_{n} * \mathrm{f}_{n}=G$, and $\left(\mathrm{H}_{n} * \mathrm{f}_{n}\right) * \mathrm{G}_{n}=G$. Hence $\mathrm{H}_{n} * \mathrm{f}_{n}=\mathrm{F}_{n}$ is the desired sequence.

Note that, in particular, the theorem holds if $\left\{G_{n}\right\}$ is a sequence of matrix-valued continuous functions and for each $n$, det $\mathrm{G}_{n} \neq 0$ on some fixed interval $[0, T]$. It is also interesting to note that the hypothesis that $\mathrm{G}_{n} \in \mathrm{S}_{T}$ for all $n$ is essential. To see this, let $\mathrm{B}$ be the Banach algebra of $2 \times 2$ matrices and define

$$
\mathrm{G}_{n}(x)=\left[\begin{array}{cc}
1 & 0 \\
0 & \mathrm{f}_{n}(x)
\end{array}\right] \text { if } n \text { is odd, } \quad \mathrm{G}_{n}(x)=\left[\begin{array}{cc}
\mathrm{f}_{n}(x) & 0 \\
0 & 1
\end{array}\right] \text { if } n \text { is even, }
$$

where

$$
\begin{aligned}
\mathrm{f}_{n}(x)=1 & \text { for } x \geqq n, \\
=0 & \text { for } 0 \leqq x<n .
\end{aligned}
$$

None of the $\mathrm{G}_{n}$ vanish on any neighborhood of the origin and each $\mathrm{G}_{n}$ has an inverse as an operator. However, one can prove directly that there is no sequence $\mathrm{F}_{n}$ and no nonzero $G$ such that $\mathrm{F}_{n} * \mathrm{G}_{n}=G$.

\section{BIBLIOGRAPHY}

1. T. K. Boehme, On sequences of continuous functions and convolution, Studia Math. 25 (1965), 333-335.

2. Arthur Erdélyi, Operational calculus and generalized functions, Holt, Rinehart and Winston, New York, 1962.

3. Jan Mikusinski, Operational calculus, Pergamon, Oxford, 1959.

GEORGETOWN UNIVERSITY 